\title{
PAIR OF COPLANAR BARENBLATT CRACKS AT THE INTERFACE OF TWO BONDED DISSIMILAR MICROPOLAR ELASTIC HALF-PLANES RAJENDRA KUMAR TRIPATHI ${ }^{1}$
}

\author{
Department of Mathematics, Pioneer Mahila Mahavidlaya, Barabanki, U.P., India
}

\section{ABSTRACT}

The condition of finiteness of stresses at the end of a crack and smooth joining of the opposite sides of the crack were first proposed in hypothetical form by Khirstianovich and proved on the basis of the principle of virtual displacement by Barenblatt.

KEYWORDS: Micropolar Poisson Ratio, Modulus of Rigidity, Classical Lame's Constant

Recent examples of such studies are proved by Sneddon (1969), Burnisten and Gurely (1973), Tresher and Smith (1973). In all these investigations however, the crack is embedded in a homogeneous medium.

\section{MATERIALS AND METHODS}

\section{Formulation of the Problem}

In this chapter, we shall study the stress and displacement field in the vicinity of a pair of coplanar Barenblatt cracks located at the interface of two bonded dissimilar micropolar elastic half planes. We consider a pair of coplanar Barenblatt cracks $a \leq|x| \leq b, y=0$ located at the interface of two bonded dissimilar micropolar elastic half planes. We suppose that two half planes $\mathrm{y}>0$ and $\mathrm{y}<0$ be occupied by elastic constants $\mu_{1}, \mathrm{k}_{1}$ and $\mu_{2}, \mathrm{k}_{2}$ with $\mathrm{k}_{\mathrm{i}}=3-4 \eta_{\mathrm{i}}(\mathrm{i}=1,2)$ where $\eta_{\mathrm{i}}$ denotes the Poison ratio of the two elastic materials and $\mu_{i}$ denotes the modulus of rigidity of two respective media.

Following Lowengrub and Sneddon (1969), we shall require that

$\mathrm{u}_{\mathrm{y}}\left(\mathrm{a}^{+}, 0+\right)=\mathrm{u}_{\mathrm{y}}\left(\mathrm{a}^{+}, 0-\right)=0$

$\mathrm{u}_{\mathrm{y}}\left(\mathrm{a}^{-}, 0+\right)=\mathrm{u}_{\mathrm{y}}\left(\mathrm{a}^{-}, 0-\right)=0$

$\mathrm{u}_{\mathrm{y}}\left(\mathrm{b}^{+}, 0+\right)=\mathrm{u}_{\mathrm{y}}\left(\mathrm{b}^{+}, 0-\right)=0$

$\mathrm{u}_{\mathrm{y}}\left(\mathrm{b}^{-}, 0+\right)=\mathrm{u}_{\mathrm{y}}\left(\mathrm{b}^{-}, 0-\right)=0$

where $y=\left(u_{x}, u_{y}, \phi\right)$. The component of stress, displacement and microrotation must satisfy the condition

$$
\begin{aligned}
& \sigma_{y y}(x, 0+)=0\left(x^{-1}\right) \\
& \sigma_{x y}(x, 0+)=0\left(x^{-1}\right), x \rightarrow \infty \\
& m_{\phi y}(x, 0+)=0\left(x^{-1}\right)
\end{aligned}
$$

If we assume that the upper and lower surface of both cracks are subjected to prescribed pressures $\mathrm{p}(\mathrm{x})$ and $q(x)$, then inside the crack following conditions are to be satisfied.

$\sigma_{\mathrm{yy}}(\mathrm{x}, 0+)=\sigma_{\mathrm{yy}}(\mathrm{x}, 0-)=-\mathrm{p}(\mathrm{x}),-\mathrm{b} \leq \mathrm{x} \leq-$

$\mathrm{a}, \mathrm{a} \leq \mathrm{x} \leq \mathrm{b}$

$\sigma_{\mathrm{xy}}(\mathrm{x}, 0+)=\sigma_{\mathrm{xy}}(\mathrm{x}, 0-)=-\mathrm{q}(\mathrm{x}),-\mathrm{b} \leq \mathrm{x} \leq-$

$\mathrm{a}, \mathrm{a} \leq \mathrm{x} \leq \mathrm{b}$

$\mathrm{m}_{\phi y}(\mathrm{x}, 0+)=\mathrm{m}_{\phi y}(\mathrm{x}, 0-)=0,-\mathrm{b} \leq \mathrm{x} \leq-\mathrm{a}, \mathrm{a} \leq \mathrm{x} \leq \mathrm{b}$

where $\mathrm{p}(\mathrm{x})$ and $\mathrm{q}(\mathrm{x})$ are the internal pressure and shear applied to the faces of the crack. For the region of the interface not occupied by the crack, following continuity conditions must be satisfied.

$\mathrm{u}_{\mathrm{x}}(\mathrm{x}, 0+)=\mathrm{u}_{\mathrm{x}}(\mathrm{x}, 0-),|\mathrm{x}|<a$, and $|\mathrm{x}|>b$,

$\mathrm{u}_{\mathrm{y}}(\mathrm{x}, 0+)=\mathrm{u}_{\mathrm{y}}(\mathrm{x}, 0-),|\mathrm{x}|<a$, and $|\mathrm{x}|>b$,

$\phi(\mathrm{x}, 0+)=\phi(\mathrm{x}, 0-),|\mathrm{x}|<a$, and $|\mathrm{x}|>b$,

$\sigma_{\mathrm{yy}}(\mathrm{x}, 0+)=\sigma_{\mathrm{yy}}(\mathrm{x}, 0-),|\mathrm{x}|<a$, and $|\mathrm{x}|>b$,

$\sigma_{\mathrm{xy}}(\mathrm{x}, 0+)=\sigma_{\mathrm{xy}}(\mathrm{x}, 0-),|\mathrm{x}|<a$, and $|\mathrm{x}|>b$,

$\mathrm{m}_{\phi \mathrm{y}}(\mathrm{x}, 0+)=\mathrm{m}_{\phi \mathrm{y}}(\mathrm{x}, 0-),|\mathrm{x}|<a$, and $|\mathrm{x}|>b$,

Following Lowengrub and Sneddon (1969), we take the displacement field-

$$
\begin{aligned}
& \mathrm{F}_{\mathrm{s}}\left[\left[\mathrm{A}_{1}-\mathrm{p}_{1} \xi^{-1} \mathrm{~B}_{1}+\mathrm{Q}_{1} \mathrm{yB}_{1}\right] \mathrm{e}^{-\xi \mathrm{y}}-\mathrm{L}_{1}^{2} \eta_{1} \mathrm{c}_{1} \mathrm{e}^{\eta_{1} \mathrm{y}}, \mathrm{y}>0\right. \\
& \mathrm{u}_{\mathrm{x}}(\mathrm{x}, \mathrm{y})= \\
& \mathrm{F}_{\mathrm{s}}\left[\mathrm{A}_{2}-\mathrm{p}_{2} \xi^{-1} \mathrm{~B}_{2}+\mathrm{Q}_{2} \mathrm{yB}_{2}\right] \mathrm{e}^{\xi \mathrm{y}}-\mathrm{L}_{2}^{2} \eta_{2} \mathrm{c}_{2} \mathrm{e}^{\eta_{2} \mathrm{y}}, \mathrm{y}<0
\end{aligned}
$$


$\mathrm{F}_{\mathrm{c}}\left[\left[\mathrm{A}_{1}+\mathrm{Q}_{1} \mathrm{yB}_{1}\right] \mathrm{e}^{-\xi \mathrm{y}}-\mathrm{L}_{1}^{2} \xi \mathrm{c}_{1} \mathrm{e}^{\eta_{1} \mathrm{y}}, \mathrm{y}>0\right.$

$\mathrm{u}_{\mathrm{y}}(\mathrm{x}, \mathrm{y})=$

$\mathrm{F}_{\mathrm{c}}\left[\mathrm{A}_{2}+\mathrm{Q}_{2} \mathrm{~B}_{2}\right] \mathrm{e}^{\xi \mathrm{y}}+\mathrm{L}_{2}^{2} \xi \mathrm{c}_{2} \mathrm{e}^{\eta_{2} \mathrm{y}}, \mathrm{y}<0(2.11)$

$F_{s}\left[\left[B_{1} e^{-\xi y}+c_{1} e^{-\eta_{1} y}\right] \quad, y>0\right.$

$\phi(\mathrm{x}, \mathrm{y})=$

$\mathrm{F}_{\mathrm{s}}\left[\mathrm{B}_{2} \mathrm{e}^{\xi \mathrm{y}}+\mathrm{c}_{2} \mathrm{e}^{-\eta_{2} \mathrm{y}}\right] \quad, \mathrm{y}<0$

where $F_{S}$ and $F_{c}$ are the Fourier sine and consine transforms. We suppose that

$P_{i}=\frac{\lambda_{i}+3 \mu_{i}}{\lambda_{i}+2 \mu_{i}}, Q_{i}=\frac{\lambda_{i}+\mu_{i}}{\lambda_{i}+2 \mu_{i}}, L_{i}^{2}=\frac{v_{i}}{2 \mu_{i}}$

$\Gamma_{1}=\frac{\mu_{1}}{2 \mu_{2}}, \Gamma_{2}=\frac{v_{1}}{v_{2}}, \quad p_{i}=2-Q_{i} i=1,2$

Here $\lambda_{i}$ and $\mu_{i}$ are the classical Lames' constants and $v_{\mathrm{i}}$ is the micropolar modulii and $\mathrm{Q}_{\mathrm{i}}$ is the micropolar poisson ration. The micropolar modulii $v_{i}$ and $\mu_{i}$ have the dimensions of force and stress respectively. The internal characteristics length $\mathrm{L}_{\mathrm{i}}$ of the medium given by

$$
L_{i}=\sqrt{\frac{v_{i}}{2 \mu_{i}}}
$$

from equations (2.1) and (2.7) we see that $\sigma_{y y}(x, 0+)=\sigma_{y y}(x, 0-)$ for all values of $x$ and it is easily shown that this condition is equivalent to the single equation

$$
\begin{aligned}
\xi \mathrm{A}_{2}+\left(1-\mathrm{Q}_{2}\right) \mathrm{B}_{2} & +\mathrm{L}_{2}^{2} \xi \mathrm{C}_{2} \\
& =\Gamma_{1}\left\{-\xi \mathrm{A}_{1}+\left(1-\mathrm{Q}_{1}\right) \mathrm{B}_{1}+\mathrm{L}_{1}^{2} \xi \eta_{1} \mathrm{C}_{1}\right\}
\end{aligned}
$$

from equations (2.2) and (2.8) we see that $\sigma_{x y}(x, 0+)=\sigma_{x y}(x, 0-)$ the boundary condition is equivalent to the single equation

$$
\xi \mathrm{A}_{2}+\left(1-\mathrm{Q}_{2}\right) \mathrm{B}_{2}+\mathrm{L}_{2}^{2} \xi^{2} \mathrm{C}_{2}=\Gamma_{1}\left\{\xi \mathrm{A}_{1}-\mathrm{B}_{1} \mathrm{~L}_{1}^{2} \xi \eta_{1} \mathrm{C}_{1}\right\}
$$

Similarly from equation (2.3) and (2.9) we see that $\mathrm{m}_{\phi y}(\mathrm{x}, 0+)=\mathrm{m}_{\phi y}(\mathrm{x}, 0-)$ for all values of $\mathrm{x}$ and it is easily shown that this condition is equivalent to the single equation

$$
\xi \mathrm{B}_{2}+\eta_{2} \mathrm{C}_{2}=\Gamma_{2}\left(\xi \mathrm{B}_{1}+\mathrm{B}_{1} \eta_{1} \mathrm{C}_{1}\right)
$$

Solving these equations for $\mathrm{A}_{2}, \mathrm{~B}_{2}, \mathrm{C}_{2}$ in terms of $\mathrm{A}_{1}, \mathrm{~B}_{1}, \mathrm{C}_{1}$ we find that

$$
\begin{aligned}
& \mathrm{A}_{2}=-\Gamma_{1}\left[1+2 \mathrm{Q}^{-1}\left\{\left(1-\mathrm{Q}_{2}\right) \eta_{2}+\eta_{2} \xi^{2} \mathrm{~L}_{2}^{2}\right\}\right] \mathrm{A}_{1} \\
& +\quad \xi^{-1}\left(\Gamma_{1}\left(1-\mathrm{Q}_{1}\right)-\mathrm{Q}^{-1}\left(1-\mathrm{Q}_{2}\right)\left\{\Gamma _ { 2 } \mathrm { L } _ { 2 } ^ { 2 } \xi ^ { 2 } \left(\eta_{2}-\right.\right.\right. \\
& \left.\xi)-\Gamma_{1} \eta_{2}\left(2-\mathrm{Q}_{2}\right)\right\} \mathrm{B}_{2}=\mathrm{Q}^{-1}\left(2 \Gamma_{1} \eta_{2} \xi \mathrm{A}_{1}+\left\{\Gamma _ { 2 } \mathrm { L } _ { 2 } ^ { 2 } \xi ^ { 2 } \left(\eta_{2}-\right.\right.\right. \\
& \left.\xi)-\Gamma_{1} \eta_{2}\left(2-\mathrm{Q}_{1}\right)\right\} \mathrm{B}_{1}+\left[\Gamma_{1} \eta_{1} \mathrm{~L}_{1}^{2}-\mathrm{Q}^{-1}((1-\right. \\
& \left.\mathrm{Q}_{1}\right)\left\{\Gamma_{1} \eta_{1} \mathrm{~L}_{2}^{2}\left(\eta_{2}-\xi\right)-\Gamma_{2} \eta_{2} \mathrm{~L}_{1}^{2}\left(\eta_{1}+\xi\right)+\eta_{2} \mathrm{~L}_{2}^{2}\left\{\Gamma _ { 1 } \xi ^ { 2 } \left(\eta_{1}+\right.\right.\right. \\
& \left.\left.\left.\xi) \mathrm{L}_{1}^{2}+\Gamma_{2} \eta_{1} \mathrm{Q}_{2}\right\}\right)\right] \mathrm{C}_{1}
\end{aligned}
$$

$\mathrm{B}_{2}=\quad \mathrm{Q}^{-1}\left[2 \Gamma_{1} \eta_{2} \xi \mathrm{A}_{1}+\left\{\Gamma_{2} \mathrm{~L}_{2}^{2} \xi^{2}\left(\eta_{2}-\xi\right)-\Gamma_{1} \eta_{2}(2-\right.\right.$ $\left.\left.\left.\mathrm{Q}_{1}\right)\right\} \mathrm{B}_{1}+\left\{\eta_{1} \mathrm{~L}_{2}^{2} \Gamma_{2} \xi\left(\eta_{2}-\xi\right)-\Gamma_{1} \eta_{2} \mathrm{~L}_{1}^{2} \xi\left(\eta_{2}+\xi\right)\right\} \mathrm{C}_{1}\right]$

$\mathrm{C}_{2}=\quad \mathrm{Q}^{-1}\left[-2 \xi^{2} \Gamma_{1} \mathrm{~A}_{1}+\left\{\Gamma_{2} \mathrm{Q}_{2} \xi+\left(2-\mathrm{Q}_{1}\right) \xi \Gamma_{1}\right\} \mathrm{B}_{1}+\right.$ $\left.\left\{\Gamma_{2} \mathrm{Q}_{2} \eta_{1}+\Gamma_{1} \mathrm{~L}_{1}^{2} \xi^{2}\left(\eta_{1}+\xi\right)\right\} \mathrm{C}_{1}\right]$

$\mathrm{Q}=\mathrm{L}_{2}^{2} \xi^{2}\left(\eta_{2}-\xi\right)+\mathrm{Q}_{2} \eta_{2}$

Now from equations $(2.10-2.12)$ we see that the boundary conditions $(2.4-2.6)$ are equivalent to the conditions

$$
\begin{aligned}
& \mathrm{F}_{\mathrm{S}}\left[\xi\left(\mathrm{A}_{1}+\mathrm{A}_{2}\right)+\left\{\left(2-\mathrm{Q}_{2}\right) \mathrm{B}_{2}-\left(2-\mathrm{Q}_{1}\right) \mathrm{B}_{1}\right\}+\mathrm{L}_{2}^{2} \xi \eta_{2} \mathrm{C}_{2}\right. \\
& \left.-\mathrm{L}_{1}^{2} \xi \eta_{1} \mathrm{C}_{1}: \mathrm{x}\right]=0 \\
& \mathrm{~F}_{\mathrm{c}}\left[\mathrm{A}_{1}-\mathrm{A}_{2}-\xi\left(\mathrm{L}_{1}^{2} \mathrm{C}_{1}+\mathrm{L}_{2}^{2} \mathrm{C}_{2}\right): \mathrm{x}\right]=0 \\
& \mathrm{~F}_{\mathrm{s}}\left[\mathrm{B}_{1}-\mathrm{B}_{2}+\mathrm{C}_{1}+\mathrm{C}_{2}: x\right]=0
\end{aligned}
$$

Substituting the values of $A_{2}, B_{2}, C_{2}$ in the above equations (2.13) and applying the boundary conditions (2.12.3) we get.

$$
\begin{gathered}
\mathrm{F}_{\mathrm{c}}\left[-\xi \mathrm{A}_{1}+\mathrm{B}_{1}+\mathrm{L}_{1}^{2} \xi^{2} \mathrm{C}_{1}: x\right]=\frac{\mathrm{p}(\mathrm{x})}{2 \mu_{1}^{\prime}} \quad \begin{array}{c}
\mathrm{b} \leq \mathrm{x} \leq-\mathrm{a} \\
\mathrm{a} \leq \mathrm{x} \leq \mathrm{b}
\end{array} \\
\mathrm{F}_{\mathrm{s}}\left[-\xi \mathrm{A}_{1}+\left(1-\mathrm{Q}_{1}\right) \mathrm{B}_{1}+\mathrm{L}_{1}^{2} \xi^{2} \mathrm{C}_{1}: x\right] \\
=\frac{\mathrm{p}(\mathrm{x})}{2 \mu_{1}^{\prime}} \quad-\mathrm{b} \leq \mathrm{x} \leq-\mathrm{a} \\
\end{gathered}
$$


$\mathrm{F}_{\mathrm{c}}\left[\mathrm{B}_{1} \xi+\eta_{1} \mathrm{C}_{1}: \mathrm{x}\right]=0 \quad \begin{gathered}-\mathrm{b} \leq \mathrm{x} \leq-\mathrm{a} \\ \mathrm{a} \leq \mathrm{x} \leq \mathrm{b}\end{gathered}$

If we now express $A_{1}, B_{1}$ and $C_{1}$ in terms of $\phi(\xi)$, $\Psi(\xi)$ and $\mathrm{X}(\xi)$ through the equations

$a A_{1}=\left(\left(b_{2} c_{3}-b_{3} c_{2}\right) c_{2} \phi(\xi)-\left\{\left(b_{2} c_{3}-b_{3} c_{2}\right) c_{1}+\left(b_{1} c_{2}-b_{2} c_{1}\right)\right.\right.$ $\left.\left.c_{3}\right\} \Psi(\xi)+\left(b_{1} c_{2}-b_{2} c_{1}\right) c_{2} X(\xi)\right)$

$\mathrm{aB}_{1}=-\left(\left(\mathrm{a}_{2} \mathrm{c}_{3}-\mathrm{a}_{3} \mathrm{c}_{2}\right) \mathrm{c}_{2} \phi(\xi)-\left\{\left(\mathrm{a}_{1} \mathrm{c}_{2}-\mathrm{a}_{2} \mathrm{c}_{1}\right) \mathrm{c}_{3}+\left(\mathrm{a}_{2} \mathrm{c}_{3}-\mathrm{a}_{3} \mathrm{c}_{2}\right)\right.\right.$ $\left.\left.c_{1}\right\} \Psi(\xi)+\left(a_{1} c_{2}-a_{2} c_{1}\right) c_{2} X(\xi)\right)$

$\mathrm{aC}_{1}=a \mathrm{c}_{1}^{-1} \phi(\xi)-\left(\mathrm{a}_{1} \mathrm{D}_{1}+\mathrm{b}_{1} \mathrm{D}_{2}\right)$

Where

$$
\begin{array}{ll}
\mathrm{a}_{1} \mathrm{~A}_{1}+\mathrm{b}_{1} \mathrm{~B}_{1} & +\mathrm{c}_{1} \mathrm{C}_{1}=\phi(\xi) \\
\mathrm{a}_{2} \mathrm{~A}_{1}+\mathrm{b}_{2} \mathrm{~B}_{1} & +\mathrm{c}_{2} \mathrm{C}_{1}=\Psi(\xi) \\
\mathrm{a}_{3} \mathrm{~A}_{1}+\mathrm{b}_{3} \mathrm{~B}_{1} & +\mathrm{c}_{3} \mathrm{C}_{1}=\mathrm{X}(\xi)
\end{array}
$$

Putting the values of $A_{1}, B_{1}, C_{1}$ in the equations (2.14) which are further reduced to the following set of equations:

$a=\left(a_{1} c_{2}-a_{2} c_{1}\right)\left(b_{2} c_{3}-b_{3} c_{2}\right)-\left(a_{2} c_{3}-a_{3} c_{2}\right)\left(b_{1} c_{2}-b_{2} c_{1}\right)$

$\mathrm{a}_{1}=1-\Gamma_{1}+2 \Gamma_{1} \mathrm{Q}^{-1}\left(\eta_{2}\left(2-\mathrm{Q}_{2}\right)-\xi^{2} \mathrm{~L}_{2}^{2} \eta_{2}-\right.$ $\left.\eta_{2}\left\{1-Q_{2}\right\}+\xi^{2} L_{2}^{2}\right)$

$$
\mathrm{b}_{1}=\mathrm{L}_{2}^{2} \eta_{2} \mathrm{Q}^{-1} \xi\left\{\Gamma_{2} \mathrm{Q}_{2}+\Gamma_{1}\left(2-\mathrm{Q}_{1}\right)\right\}+\xi^{-1} \Gamma_{1}\left(1-\mathrm{Q}_{1}\right)
$$

$$
\begin{aligned}
-Q^{-1}\left[\left(1-Q_{2}\right)\{\right. & \left.\Gamma_{2} L_{2}^{2} \xi^{2}\left(\eta_{2}-\xi\right)-\Gamma_{1} \eta_{2}\left(2-Q_{1}\right)\right\} \\
& \left.+\xi^{2} L_{2}^{2} \eta_{2}\left\{\Gamma_{1}\left(2-Q_{1}\right)+\Gamma_{2} Q_{2}\right\}\right] \\
& +\left(2-Q_{2}\right) \xi^{-1} Q^{-1}\left\{\Gamma_{2} L_{2}^{2} \xi^{2}\left(\eta_{2}-\xi\right)\right. \\
& \left.-\Gamma_{1} \eta_{2}\left(2-Q_{1}\right)\right\}-\xi^{-1}\left(2-Q_{1}\right)
\end{aligned}
$$

$$
\begin{aligned}
c_{1}=\Gamma_{1} \eta_{1} L_{1}^{2}-Q^{-1} & {\left[( 1 - Q _ { 2 } ) \left\{\eta_{1} \Gamma_{1} L_{2}^{2}\left(\eta_{2}-\xi\right)\right.\right.} \\
& \left.-\Gamma_{2} \eta_{2} L_{1}^{2}\left(\eta_{2}+\xi\right)\right\} \\
& \left.+\eta_{2} L_{2}^{2}\left\{\Gamma_{1} \xi^{2}\left(\eta_{1}+\xi\right) L_{1}^{2}+\Gamma_{2} \eta_{1} Q_{2}\right\}\right] \\
& +\left(2-Q_{2}\right) Q^{-1}\left\{\eta_{1} \Gamma_{2} L_{2}^{2}\left(\eta_{2}-\xi\right)\right\}
\end{aligned}
$$

$$
\begin{aligned}
\left.-\Gamma_{1} \eta_{1} L_{1}^{2} \xi\left(\eta_{1}+\xi\right)\right) & \\
& +L_{2}^{2} \eta_{2} Q^{-1}\left\{\Gamma_{2} Q_{2} \eta_{1}+\Gamma_{1} \xi^{2}\left(\eta_{1}+\xi\right)\right. \\
& \left.-L_{1}^{2} \eta_{1}\right\}
\end{aligned}
$$$$
\mathrm{a}_{2}=1+\Gamma_{1}+2 \mathrm{Q}^{-1}\left(\Gamma_{1} \eta_{2}\left(1-\mathrm{Q}_{2}\right)+\Gamma_{1} \eta_{2} \xi^{2} \mathrm{~L}_{2}^{2}+\xi^{3} \mathrm{~L}_{2}^{2}\right.
$$$$
\mathrm{a}_{3}=-2 \Gamma_{1} \xi Q^{-1}\left(\eta_{2}+\xi\right)
$$

$$
\begin{aligned}
\mathrm{b}_{2}=\mathrm{Q}^{-1}\left[\left(1-\mathrm{Q}_{2}\right)\right. & \left\{\Gamma_{2} \Gamma_{2}^{2} \xi\left(\eta_{2}-\xi\right)-\Gamma_{1} \xi^{-1} \eta_{2}\left(2-\mathrm{Q}_{1}\right)\right\} \\
& +\mathrm{L}_{2}^{2} \eta_{2}\left\{\Gamma_{1} \xi\left(2-\mathrm{Q}_{1}\right)+\Gamma_{2} \xi \mathrm{Q}_{2}\right\} \\
& \left.+\mathrm{L}_{2}^{2} \xi^{2}\left\{\Gamma_{2} \mathrm{Q}_{2}+\left(2-\mathrm{Q}_{1}\right) \Gamma_{1}\right\}\right] \\
& -\xi^{-1} \Gamma_{1}\left(1-\mathrm{Q}_{1}\right) \\
& \\
\mathrm{c}_{2}=\mathrm{Q}^{-1}\left[\left(1-\mathrm{Q}_{2}\right)\right. & \left\{\Gamma_{1} \eta_{1} \mathrm{~L}_{2}^{2}\left(\eta_{2}-\xi\right)-\Gamma_{2} \eta_{2} \mathrm{~L}_{1}^{2}\left(\eta_{1}+\xi\right)\right. \\
& \left.+\eta_{2} \mathrm{~L}_{2}^{2}\right\}\left\{\Gamma_{1} \xi^{2}\left(\eta_{1}+\xi\right) \mathrm{L}_{1}^{2}+\Gamma_{2} \eta_{1} \mathrm{Q}_{2}\right\} \\
& +\mathrm{L}_{2}^{2} \xi\left\{\Gamma_{2} \mathrm{Q}_{2} \eta_{1}+\Gamma_{1} \mathrm{~L}_{1}^{2} \xi^{2}\left(\eta_{1}+\xi\right)\right\} \\
& \left.+\mathrm{L}_{1}^{2} \xi-\Gamma_{1} \eta_{1} \mathrm{~L}_{1}^{2}\right]
\end{aligned}
$$

$$
\begin{aligned}
& \mathrm{a}_{3}=-2 \Gamma_{1} \xi \mathrm{Q}^{-1}\left(\eta_{2}+\xi\right) \\
& \begin{array}{r}
\mathrm{b}_{3}=1+\mathrm{Q}^{-1}\left[\Gamma_{2} \mathrm{Q}_{2} \xi\left(2-\mathrm{Q}_{1}\right) \xi \Gamma_{1}\right. \\
\left.\quad-\left\{\Gamma_{2} \mathrm{~L}_{2}^{2} \xi^{2}\left(\eta_{2}-\xi\right)-\Gamma_{1} \eta_{2}\left(2-\mathrm{Q}_{1}\right)\right\}\right]
\end{array} \\
& \begin{array}{c}
\mathrm{c}_{3}=1+\mathrm{Q}^{-1}\left[\Gamma_{2} \mathrm{Q}_{2} \eta_{1}+\Gamma_{1} \mathrm{~L}_{1}^{2} \xi^{2}\left(\eta_{1}+\xi\right)\right. \\
\left.-\xi\left\{\Gamma_{2} \eta_{1} \mathrm{~L}_{2}^{2}\left(\eta_{2}-\xi\right)-\Gamma_{1} \eta_{2}\left(\eta_{1}+\xi\right)\right\}\right] \\
\mathrm{D}_{1}=\left(\mathrm{b}_{2} \mathrm{c}_{3}-\mathrm{b}_{3} \mathrm{c}_{2}\right) \mathrm{c}_{2} \phi(\xi)-\left\{\left(\mathrm{b}_{2} \mathrm{c}_{3}-\mathrm{b}_{3} \mathrm{c}_{2}\right) \mathrm{c}_{1}+\left(\mathrm{b}_{1} \mathrm{c}_{2}-\mathrm{b}_{2} \mathrm{c}_{1}\right)\right. \\
\left.\left.\mathrm{c}_{3}\right\} \psi(\xi)+\left(\mathrm{b}_{1} \mathrm{c}_{2}-\mathrm{b}_{2} \mathrm{c}_{1}\right) \mathrm{c}_{2} \mathrm{X}(\xi)\right\}
\end{array}
\end{aligned}
$$




$$
0,0<\mathrm{x}<\mathrm{a}, \mathrm{x}>\mathrm{b}
$$

$D_{2}=\left(a_{3} c_{2}-a_{2} c_{3}\right) c_{2} \phi(\xi)+\left\{\left(a_{1} c_{2}-a_{2} c_{1}\right) c_{3}+\left(a_{2} c_{3}-a_{3} c_{2}\right)\right.$ $\left.\mathrm{c}_{1}\right\} \psi(\xi)$

$-\left(a_{1} c_{2}-a_{2} c_{1}\right) c_{2} X(\xi)$

Putting the values of $A_{1}, B_{1}, C_{1}$ in the equations (2.14) which are further reduced to the following set of equations:

$\mathrm{F}_{\mathrm{c}}(\mathrm{a}(\xi) \phi(\xi)+\mathrm{b}(\xi) \Psi(\xi)+\mathrm{c}(\xi) \mathrm{X}(\xi): \mathrm{x})=\mathrm{f}_{1}(\mathrm{x}), \mathrm{a} \leq \mathrm{x}$ $\leq \mathrm{b}$

$\mathrm{F}_{\mathrm{s}}(\mathrm{b}(\xi) \phi(\xi)+\mathrm{c}(\xi) \Psi(\xi)+\mathrm{a}(\xi) \mathrm{X}(\xi): \mathrm{x})=\mathrm{f}_{2}(\mathrm{x}), \mathrm{a} \leq \mathrm{x}$ $\leq \mathrm{b}$

$\mathrm{F}_{\mathrm{c}}(\mathrm{c}(\xi) \phi(\xi)+\mathrm{a}(\xi) \Psi(\xi)+\mathrm{b}(\xi) \mathrm{X}(\xi): \mathrm{x})=0, \mathrm{a} \leq \mathrm{x} \leq \mathrm{b}$

Where

$\mathrm{a}(\xi)=\mathrm{a}^{-1} \mathrm{c}_{2}\left\{\left(\mathrm{a}_{2} \mathrm{c}_{3}-\mathrm{a}_{3} \mathrm{c}_{2}\right)-\xi\left(\mathrm{b}_{2} \mathrm{c}_{3}-\mathrm{b}_{3} \mathrm{c}_{2}\right)\right\}+\mathrm{L}_{1}^{2} \xi^{2} \mathrm{c}_{1}^{-1}$

$\mathrm{b}(\xi)=\mathrm{a}^{-1}\left\{\left(\mathrm{a}_{2} \mathrm{c}_{3}-\mathrm{a}_{3} \mathrm{c}_{2}\right)+\xi\left(\mathrm{b}_{2} \mathrm{c}_{3}-\mathrm{b}_{3} \mathrm{c}_{2}\right)\right\} \mathrm{c}_{1}$

$$
+\mathrm{a}^{-1}\left\{\left(\mathrm{a}_{1} \mathrm{c}_{2}-\mathrm{a}_{2} \mathrm{c}_{1}\right)+\xi\left(\mathrm{b}_{1} \mathrm{c}_{2}-\mathrm{b}_{2} \mathrm{c}_{1}\right)\right\} \mathrm{c}_{3}
$$

$\mathrm{c}(\xi)=\mathrm{a}^{-1}\left\{\left(\mathrm{a}_{2} \mathrm{c}_{1}-\mathrm{a}_{1} \mathrm{c}_{2}\right)-\xi\left\{\left(\mathrm{b}_{1} \mathrm{c}_{2}-\mathrm{b}_{2} \mathrm{c}_{1}\right)\right\} \mathrm{c}_{2}\right.$

$\mathrm{f}_{1}(\mathrm{x})=\frac{\mathrm{aP}(\mathrm{x})}{2 \mu_{1}}$

$f_{2}(x)=\frac{a q(x)}{2 \mu_{1}}$

And

$\mathrm{F}_{\mathrm{S}}(\phi(\xi): \mathrm{x})=0, \mathrm{x}>\mathrm{b}$

$\mathrm{F}_{\mathrm{c}}(\Psi(\xi): \mathrm{x})=0, \mathrm{x}>\mathrm{b}$

$\mathrm{F}_{\mathrm{s}}(\mathrm{X}(\xi): \mathrm{x})=0, \mathrm{x}>\mathrm{b}$

We proceed as in (5) and we define.

$\mathrm{F}_{\mathrm{c}}(\phi(\xi): \mathrm{x})=0\left\{\begin{array}{l}\mathrm{r}_{1}(\mathrm{x}), \mathrm{a} \leq \mathrm{x} \leq \mathrm{b} \\ 0,0<\mathrm{x}<\mathrm{a}, \mathrm{x}>\mathrm{b}\end{array}\right.$

$\mathrm{F}_{\mathrm{s}}(\Psi(\xi): \mathrm{x})=0 \overbrace{}^{\mathrm{S}_{1}(\mathrm{x}), \mathrm{a} \leq \mathrm{x} \leq \mathrm{b}}$
$F_{s}(X(\xi): x)=0\left\{\begin{array}{l}\mathrm{w}_{1}(x), a \leq x \leq b \\ 0,0<x<a, x>b\end{array}\right.$

It is easily shown that if we make extensions $r(u)$, $\mathrm{s}(\mathrm{u})$ and $\mathrm{w}(\mathrm{u})$ of $\mathrm{r}_{1}(\mathrm{u}), \mathrm{s}_{1}(\mathrm{u})$ and $\mathrm{w}_{1}(\mathrm{u})$ to $-\mathrm{b} \leq \mathrm{x} \leq-\mathrm{a}$ as follows:

$r(u)=\left\{\begin{array}{l}r_{1}(u), a \leq u \leq b \\ r_{1}(-u),-b \leq u \leq-a\end{array}\right.$

$\mathrm{s}(\mathrm{u})=\left\{\begin{array}{l}\mathrm{s}_{1}(\mathrm{u}), \mathrm{a} \leq \mathrm{u} \leq \mathrm{b} \\ \mathrm{s}_{1}(-\mathrm{u}),-\mathrm{b} \leq \mathrm{u} \leq-\mathrm{a}\end{array}\right.$

$\mathrm{w}(\mathrm{u})=\left(\begin{array}{l}\mathrm{w}_{1}(\mathrm{u}), \mathrm{a} \leq \mathrm{u} \leq \mathrm{b} \\ \mathrm{w}_{1}(-\mathrm{u}),-\mathrm{b} \leq \mathrm{u} \leq-\mathrm{a}\end{array}\right.$

then

$\mathrm{F}_{\mathrm{S}}(\phi(\xi): \mathrm{x})=\frac{1}{\pi} \int \frac{\mathrm{r}(\mathrm{u})}{\mathrm{x}-\mathrm{u}} \mathrm{du}$

$\mathrm{F}_{\mathrm{c}}(\Psi(\xi): \mathrm{x})=\frac{1}{\pi} \int \frac{\mathrm{s}(\mathrm{u})}{\mathrm{x}-\mathrm{u}} \mathrm{du}$

$\mathrm{F}_{\mathrm{s}}(\mathrm{X}(\xi): \mathrm{x})=\frac{1}{\pi} \int \frac{\mathrm{w}(\mathrm{u})}{\mathrm{x}-\mathrm{u}} \mathrm{du}$

where $\mathrm{L}=((-\mathrm{b},-\mathrm{a}) \mathrm{U}(\mathrm{a}, \mathrm{b}))$

In like manner it is a simple matter to verify that

$\mathrm{F}_{\mathrm{c}}(\phi(\xi): \mathrm{x})=0$ 


$$
0,0<\mathrm{x}<\mathrm{a}, \mathrm{x}>\mathrm{b}
$$

where $\mathrm{s}_{1}(\mathrm{x})=\int_{\mathrm{x}}^{\mathrm{b}} \mathrm{s}_{1}(\mathrm{u}) \mathrm{du}$,

$\mathrm{F}_{\mathrm{s}}(\phi \Psi(\xi): \mathrm{x})=0$

$$
0,0<\mathrm{x}<\mathrm{a}, \mathrm{x}>\mathrm{b}
$$

where $r_{1}(x)=\int_{x}^{b} r_{1}(u) d u$

$F_{c}(X(\xi): x)=\frac{1}{\pi} \int_{a}^{X} d x \int_{L} \frac{w(u)}{u-x} d u$

If we substitute (2.17), (2.18) in the equation (2.15), we see that $r, s, w$ must be solutions to the set of singular integral equations.

$a(\xi) r(x)-\frac{b(\xi)}{\pi} \int \frac{s(u)}{L-x} d u+c(\xi) w(x)=f_{1}(x), a \leq|x| \leq b$

$\mathrm{a}(\xi) \mathrm{s}(\mathrm{x})+\frac{\mathrm{b}(\xi)}{\pi} \int \frac{\mathrm{w}(\mathrm{u})}{\mathrm{u}-\mathrm{x}} \mathrm{du}+\mathrm{c}(\xi) \mathrm{s}(\mathrm{x})=\mathrm{f}_{2}(\mathrm{x}), \mathrm{a} \leq|\mathrm{x}| \leq \mathrm{b}$

$\mathrm{a}(\xi) \mathrm{w}(\mathrm{x})-\frac{\mathrm{b}(\xi)}{\pi} \int \mathrm{L} \frac{\mathrm{r}(\mathrm{u})}{\mathrm{u}-\mathrm{x}} \mathrm{du}+\mathrm{c}(\xi) \mathrm{s}(\mathrm{x})=0, \mathrm{a} \leq|\mathrm{x}| \leq \mathrm{b}$

where $f_{1}(x)$ and $f_{2}(x)$ are even functions defined on $\mathrm{L}$. The substitution

$$
\lambda(u)=s(u)-i r(u)+w(u)
$$

reduce the pair of equations (2.20) to the single integral equation

$\mathrm{a}(\xi) \lambda(\mathrm{x})+\frac{\mathrm{c}(\xi)-\mathrm{b}(\xi))}{\pi \mathrm{i}} \mathrm{L} \frac{\lambda(\mathrm{u})}{\mathrm{x}-\mathrm{u}} \mathrm{du}=\mathrm{f}(\mathrm{x}), \mathrm{x} \in \mathrm{L} \quad$ (2.22)

where

$\mathrm{L}=((-b,-a) \mathrm{U}(\mathrm{a}, \mathrm{b}))$,

$F(x)=i f_{1}(x)+f_{2}(x)$

If we now define

$$
\left.\Lambda(\mathrm{z})=\frac{1}{2 \pi \mathrm{i}} \int \frac{\lambda(\mathrm{u})}{\mathrm{L}} \mathrm{u}-\mathrm{z}\right) \mathrm{du}
$$

then using plemelj formulae.
$\Lambda^{+}(\mathrm{x})-\Lambda^{-}(\mathrm{x})=\lambda(\mathrm{x}), \Lambda^{+}(\mathrm{x})+\Lambda^{-}(\mathrm{x})=\frac{1}{\mathrm{xi}}-\int_{\mathrm{L}} \frac{\lambda(\mathrm{u})}{(\mathrm{u}-\mathrm{x})} \mathrm{du}$,

shows that (2.22) is equivalent to the condition

$\Lambda^{+}(\mathrm{x})=-\mathrm{K} \Lambda^{-}(\mathrm{x})-\{\mathrm{c}(\varepsilon)-\mathrm{b}(\varepsilon)+\mathrm{a}(\varepsilon)\}^{-1} \mathrm{f}(\mathrm{x}), \mathrm{x} \varepsilon \mathrm{L}$

Where

$$
\mathrm{K}=\left(\frac{\mathrm{c}(\xi)-\mathrm{b}(\xi)-\mathrm{a}(\xi)}{\mathrm{c}(\xi)-\mathrm{b}(\xi)+\mathrm{a}(\xi)}\right)>0
$$

Thus, we must find a sectionally holomorphic function $\Lambda(z)$, vanishing at infinity and satisfying the condition (2.23). The solution to this problem is well known (cf p. 450 (6) and is given by

$\Lambda(\mathrm{z})=\frac{\mathrm{X}(\mathrm{z})}{2 \pi \mathrm{i}\{\mathrm{c}(\varepsilon)-\mathrm{b}(\varepsilon)-\mathrm{a}(\mathrm{\varepsilon})\}}\left[\int_{\mathrm{L}}^{\int} \frac{\mathrm{f}(\mathrm{t})}{\mathrm{K}^{+}(\mathrm{t})(\mathrm{t}-\mathrm{z})} \mathrm{dt}+\mathrm{P}(\mathrm{z}) \mathrm{X}(\mathrm{z})\right](2.24)$ where $\mathrm{P}(\mathrm{z})=\mathrm{h}_{1} \mathrm{z}+\mathrm{h}_{2}, \mathrm{~h}_{1}$ and $\mathrm{h}_{2}$ are arbitrary complex constants and $\mathrm{X}(\mathrm{z})$ is the solution to the homogeneous Riemann boudary value problem.

$X^{+}(t)=-K^{-}(t), t \varepsilon L$

The homogeneous Riemann problem is known to have a solution (p.450) (3) given by

$x(z)=[(z-a)(z+b)]^{i \omega} \cdot[(z+a)(z-b)]^{-i \omega-1 / 2}$

where

$$
\omega=\frac{1}{2 \pi} \log \left\{\frac{\mathrm{c}(\xi)-\mathrm{b}(\xi)-\mathrm{a}(\xi)}{\mathrm{c}(\xi)-\mathrm{b}(\xi)+\mathrm{a}(\xi)}\right\}
$$

In the case in which $\mathrm{f}$ is a polynomial

$\int \frac{\mathrm{f}(\mathrm{t})}{\mathrm{L} \mathrm{X}^{+}(\mathrm{t})(\mathrm{t}-\mathrm{z})}=\frac{\pi \mathrm{i}\{\mathrm{c}(\xi)-\mathrm{b}(\xi)+\mathrm{a}(\xi)\}}{\mathrm{c}(\xi)-\mathrm{b}(\xi)}\left[\frac{\mathrm{f}(\mathrm{z})}{\mathrm{X}(\mathrm{t})}-\mathrm{L}(\mathrm{z})\right]$

Where

$L(z)=\frac{1}{2 \pi} \operatorname{Lim}_{R \rightarrow \infty} \int_{0}^{2 \pi} \frac{f\left(\operatorname{Re}^{i \theta}\right) R e^{i \theta} d \theta}{x\left(R^{i \theta}\right)\left(R^{i \theta}-z\right)}$

Hence (2.214) yields

$\Lambda(\mathrm{z})=\frac{1}{2 \mathrm{~b}(\xi)}[\mathrm{f}(\mathrm{z})-\mathrm{X}(\mathrm{z}) \mathrm{L}(\mathrm{z})]+\mathrm{P}(\mathrm{z}) \mathrm{X}(\mathrm{z})$ 
for $0<\mathrm{x}<\mathrm{a}$ and $\mathrm{x}>\mathrm{b}$

$\sigma_{\text {yy }}(\mathrm{x}, 0+)=\sigma_{\text {yy }}(\mathrm{x}, 0-)=-\frac{2 \mu_{1} \mathrm{~b}(\xi)}{\mathrm{f}_{0}} \mathrm{I}_{\mathrm{m}} \Lambda^{+}(\mathrm{x})$

$\sigma_{\mathrm{xy}}(\mathrm{x}, 0+)=\sigma_{\mathrm{xy}}(\mathrm{x}, 0-)=-\frac{2 \mu_{1} \mathrm{~b}(\xi)}{\mathrm{f}_{0}} \operatorname{Re} \Lambda^{+}(\mathrm{x})$

$\mathrm{m}_{\phi y}(\mathrm{x}, 0+)=\mathrm{m}_{\phi \mathrm{y}}(\mathrm{x}, 0-)=\frac{2 \mu_{1} \mathrm{~b}(\xi)}{\mathrm{f}_{0}} \mathrm{I}_{\mathrm{m}} \Lambda^{+}(\mathrm{x})$

\section{The Case of Constant Internal Pressure}

We now consider the case in which the cracks are opened by constant normal and shearing pressure say $\mathrm{p}(\mathrm{x})=$ $\mathrm{q}(\mathrm{x})=\mathrm{P}_{0}$ so that,

$$
f(x)=\frac{a P_{0}}{2 \mu_{1}}=f_{0}
$$

Thus, if $\beta=1 / 2-i \omega$, then

$$
\begin{aligned}
& \int_{0}^{2 \pi} \frac{f\left(\operatorname{Re}^{\mathrm{i} \theta}\right) R \mathrm{e}^{\mathrm{i} \theta} \mathrm{d} \theta}{\mathrm{X}\left(\mathrm{Re}^{\mathrm{i} \theta}\right)\left(\mathrm{Re}^{\mathrm{i} \theta}-\mathrm{z}\right)}=2 \pi \mathrm{f}_{0}\left\{\mathrm{z}^{2}+2 \mathrm{bz} \beta-2 \mathrm{a} \beta \mathrm{z}-\mathrm{bz}+\mathrm{az}-\right. \\
& \left.(\beta-1) \frac{5 \mathrm{~b}^{2}+4 \mathrm{a}^{2}}{2}+\left(4 \beta^{2}+4 \beta-1\right)\right\} \mathrm{C}\left(\mathrm{R}^{-1}\right)
\end{aligned}
$$

So that

$L(z)=f_{0}\left[z^{2}+(2 \beta-1)(b-a) z-\beta(\beta-1) \frac{4 a^{2}+5 b^{2}}{2}+\right.$

$$
\cdots]
$$

If follows from (2.29) that

$$
\Lambda(\mathrm{z})=\frac{\mathrm{if}_{0}}{2 \mathrm{a}(\xi) \mathrm{z}}\left[1-\left\{\mathrm{z}^{2}+\mathrm{h}_{1} \mathrm{z}+\mathrm{h}_{2}\right\} \mathrm{X}(\mathrm{z})\right]
$$

where $X(z)$ is already defined by the equation (2.26) and $h_{1}, h_{2}$ are arbitrary complex constants.

We obtain relation (2.26), the expressions for $\mathrm{X}^{+}$and $\mathrm{X}^{-}$as follows.

$X^{+}(x)=-i K^{1 / 2}\left(\left(x^{2}-a^{2}\right)\left(b^{2}-x^{2}\right)\right)^{-1 / 2}(\cos \omega \theta+i \sin \omega \theta)$

$\mathrm{X}^{-}(\mathrm{x})=\mathrm{iK}^{-1 / 2}\left(\left(\mathrm{x}^{2}-\mathrm{a}^{2}\right)\left(\mathrm{b}^{2}-\mathrm{x}^{2}\right)\right)^{-1 / 2}(\cos \omega \theta+\mathrm{i} \sin \omega \theta)$

Where

$$
\theta=\log \left\{\frac{(x-a)(x+b)}{(x+a)(b-x)}\right\}, \text { while }
$$

(i) for - b $<x<-a$,
$X^{+}(x)=i K^{1 / 2}\left(\left(x^{2}-a^{2}\right)\left(b^{2}-x^{2}\right)\right)^{-1 / 2}(\cos \omega \theta+i \sin \omega \theta)$

$\mathrm{X}^{-}(\mathrm{x})=-\mathrm{iK}^{1 / 2}\left(\left(\mathrm{x}^{2}-\mathrm{a}^{2}\right)\left(\mathrm{b}^{2}-\mathrm{x}^{2}\right)\right)^{-1 / 2}(\cos \omega \theta+\mathrm{i} \sin \omega \theta)$

(ii) for $0<\mathrm{x}<\mathrm{a}$

$\mathrm{X}^{+}(\mathrm{x})=\mathrm{X}^{-}(\mathrm{x})=-\left(\left(\mathrm{a}^{2}-\mathrm{x}^{2}\right)\left(\mathrm{b}^{2}-\mathrm{x}^{2}\right)\right)^{-1 / 2}\left(\cos \omega \theta_{1}+\mathrm{i}\right.$ $\left.\sin \omega \theta_{1}\right)$

Where

$\theta_{1}=\log \left\{\frac{(a-x)(b+x)}{(a+x)(b-x)}\right\}$

(iii) for $\mathrm{x}>\mathrm{b}$

$\mathrm{X}^{+}(\mathrm{x})=\mathrm{X}^{-}(\mathrm{x})=\left(\left(\mathrm{x}^{2}-\mathrm{a}^{2}\right)\left(\mathrm{x}^{2}-\mathrm{b}^{2}\right)\right)^{-1 / 2}\left(\cos \omega \theta_{2}+\mathrm{i} \sin \right.$ $\left.\omega \theta_{2}\right)$

Where

$\theta_{2}=\log \left\{\frac{(x-a)(x+b)}{(x+a)(x-b)}\right\}$

Hence for $\mathrm{a}<\mathrm{x}<\mathrm{b}$, we find that if we suppose

$$
\mathrm{h}_{1}=\mathrm{h}_{1}^{1}+\mathrm{ih} \mathrm{h}_{1}^{2} \mathrm{~h}_{2}=\mathrm{h}_{2}^{1}+\mathrm{ih}_{2}^{2}
$$

then,

$$
\begin{aligned}
& \Lambda^{+}(x)-\Lambda^{-}(x)=\frac{F_{0}}{\sqrt{\left\{b(\xi)^{2}-a(\xi)^{2}\right\}}}\left(( x ^ { 2 } - a ^ { 2 } ) \left(b^{2}-\right.\right. \\
& \left.\left.x^{2}\right)\right)^{-1 / 2}\left\{\left(x^{2}+h_{1}^{1} x+h_{2}^{1}\right)\right. \\
& \left.. \cos \omega \theta^{\prime}\left(h_{1}^{2} x+h_{2}^{2}\right) \sin \omega \theta\right\}+i\left(\left(h_{1}^{2} x+h_{2}^{2}\right)\right. \\
& \left.. \cos \omega \theta+\left(x^{2}+h_{1}^{1} x+h_{2}^{1}\right) \sin \omega \theta\right)
\end{aligned}
$$

While

$$
\begin{gathered}
\begin{aligned}
\Lambda^{+}(\mathrm{x})+\Lambda^{-}(\mathrm{x})= & \frac{\mathrm{F}_{0} \mathrm{~b}(\xi)}{\mathrm{a}(\xi) \sqrt{\mathrm{a}(\xi)^{2}-\mathrm{b}(\xi)^{2}}}\left(( \mathrm { x } ^ { 2 } - \mathrm { a } ^ { 2 } ) \left(\mathrm{~b}^{2}\right.\right. \\
& \left.\left.-\mathrm{x}^{2}\right)\right)^{-1 / 2}
\end{aligned} \\
\left\{\left(\mathrm{x}^{2}+\mathrm{h}_{1}^{1} \mathrm{x}+\mathrm{h}_{2}^{1}\right) \cos \omega \theta-\left(\mathrm{h}_{1}^{2} \mathrm{x}+\mathrm{h}_{2}^{2}\right) \sin \omega \theta\right\}
\end{gathered}
$$

$+i\left(\frac{f_{0}}{a(\xi)}+\frac{f_{0} b(\xi)}{a(\xi) \sqrt{a(\xi)^{2}-b(\xi)^{2}}}\left[\left(x^{2}-a^{2}\right)\left(b^{2}-x^{2}\right)\right]^{-1 / 2}\right.$

$\left.\left\{\left(x^{2}+h_{1}^{1} x+h_{2}^{2}\right) \sin \omega \theta+\left(h_{1}^{2} x+h_{2}^{2}\right) \cos \omega \theta\right\}\right)$ 
On $\mathrm{a}<\mathrm{x}<\mathrm{b}$, the Plemelj relation yield,

$\mathrm{s}_{1}(\mathrm{x})=\frac{-\mathrm{f}_{0}}{\sqrt{\mathrm{a}(\xi)^{2}-\mathrm{b}(\xi)^{2}}}\left(\left(\mathrm{x}^{2}-\mathrm{a}^{2}\right)\left(\mathrm{b}^{2}-\mathrm{x}^{2}\right)\right)^{-1 / 2}\left\{\left(\mathrm{x}^{2}+\mathrm{h}_{1}^{2} \mathrm{x}+\right.\right.$ $\left.\left.\left.h_{2}^{1}\right) \cos \omega \theta+\left(h_{1}^{2} x+h_{2}^{2}\right) \cdot \sin \omega \theta\right\}\right)$

$r_{1}(x)=\frac{-f_{0}}{\sqrt{a(\xi)^{2}-b(\xi)^{2}}}\left(\left(x^{2}-a^{2}\right)\left(b^{2}-x^{2}\right)\right)^{-1 / 2} \quad\left\{\left(\quad h_{1}^{1} x+\right.\right.$ $\left.\left.\left.h_{2}^{2}\right) \cos \omega \theta-\left(x^{2}+h_{1}^{1} x+h_{2}^{1}\right) \cdot \sin \omega \theta\right\}\right)$

$\mathrm{w}_{1}(\mathrm{x})=\frac{\mathrm{f}_{0}}{\sqrt{\mathrm{a}(\xi)^{2}-\mathrm{b}(\xi)^{2}}}\left(\left(\mathrm{x}^{2}-\mathrm{a}^{2}\right)\left(\mathrm{b}^{2}-\mathrm{x}^{2}\right)\right)^{-1 / 2}\left\{\left(\mathrm{x}^{2}+\mathrm{h}_{1}^{1} \mathrm{x}+\right.\right.$ $\left.\left.\left.h_{2}^{1}\right) \cos \omega \theta+\left(h_{1}^{2} x+h_{2}^{2}\right) \cdot \sin \omega \theta\right\}\right)$

we may also note on $-\mathrm{b}<\mathrm{x}<-\mathrm{a}$

$s(x)=\frac{f_{0}}{\sqrt{a(\xi)^{2}-b(\xi)^{2}}}\left(\left(x^{2}-a^{2}\right)\left(b^{2}-x^{2}\right)\right)^{-1 / 2}\left\{\left(x^{2}+h_{1}^{1} x+\right.\right.$ $\left.\left.\mathrm{h}_{2}^{1}\right) \cos \omega \theta-\left(\mathrm{h}_{1}^{2} \mathrm{x}+\mathrm{h}_{2}^{1}\right) \cdot \sin \omega \theta\right\}$

$r(x)=\frac{-f_{0}}{\sqrt{a(\xi)^{2}-b(\xi)^{2}}}\left(\left(x^{2}-a^{2}\right)\left(b^{2}-x^{2}\right)\right)^{-1 / 2}\left\{\left(x^{2}+h_{1}^{1} x+\right.\right.$ $\left.\left.h_{2}^{1}\right) \sin \omega \theta-\left(h_{1}^{2} x+h_{2}^{2}\right) \cdot \cos \omega \theta\right\}$

$\mathrm{w}(\mathrm{x})=\frac{-\mathrm{f}_{0}}{\sqrt{\mathrm{a}(\xi)^{2}-\mathrm{b}(\xi)^{2}}}\left(\left(\mathrm{x}^{2}-\mathrm{a}^{2}\right)\left(\mathrm{b}^{2}-\mathrm{x}^{2}\right)\right)^{-1 / 2}\left\{\left(\mathrm{x}^{2}+\mathrm{h}_{1}^{1} \mathrm{x}+\right.\right.$ $\left.\left.h_{2}^{1}\right) \cos \omega \theta+\left(h_{1}^{2} x+h_{2}^{2}\right) \cdot \sin \omega \theta\right\}$

Hence, the relation $s(x)=-s_{1}(-x), \quad r(x)=r_{1}(-x)$ and $\mathrm{w}(\mathrm{x})=\mathrm{w}_{1}(-\mathrm{x})$ on $-\mathrm{b}<\mathrm{x}<-\mathrm{a}$ be satisfied, we must choose $h_{1}^{1}$ and $h_{2}^{2}$ so that $h_{1}^{1}=h_{2}^{2}=0$.

Another use of the Plemelj formulae yield

$\frac{1}{\pi} \int \frac{\mathrm{r}(\mathrm{u})}{\mathrm{L}-\mathrm{u})} \mathrm{du}=\frac{\mathrm{f}_{0} \mathrm{~b}(\xi)}{\mathrm{a}(\xi) \sqrt{\mathrm{a}(\xi)^{2}-\mathrm{b}(\xi)^{2}}}\left(\left(\mathrm{x}^{2}-\mathrm{a}^{2}\right)\left(\mathrm{b}^{2}-\mathrm{x}^{2}\right)\right)^{-1 / 2}$

$\left\{\left(\mathrm{x}^{2}+\mathrm{h}_{2}^{1}\right) \cos \omega \theta-\mathrm{h}_{1}^{2} \mathrm{x} \sin \omega \theta\right\}$

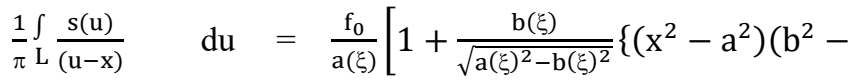
$\left.\left.\left.x^{2}\right)\right\}\right]^{-1 / 2}$

$\left\{\left(\mathrm{x}^{2}+\mathrm{h}_{2}^{1}\right) \sin \omega \theta-\mathrm{h}_{1}^{2} \mathrm{x} \cos \omega \theta\right\}$ $\left.\frac{1}{\pi} \int \frac{\mathrm{w}(\mathrm{u})}{\mathrm{L}} \quad \mathrm{du}-\mathrm{x}\right) \quad=\frac{-\mathrm{f}_{0}}{\mathrm{a}(\xi)}\left[1+\frac{\mathrm{b}(\xi)}{\sqrt{\mathrm{a}(\xi)^{2}-\mathrm{b}(\xi)^{2}}}\left\{\left(\mathrm{x}^{2}-\mathrm{a}^{2}\right)\left(\mathrm{b}^{2}-\right.\right.\right.$ $\left.\left.\left.x^{2}\right)\right\}\right]^{-1 / 2}$

$\left\{\left(\mathrm{x}^{2}+\mathrm{h}_{2}^{1}\right) \sin \omega \theta-\mathrm{h}_{1}^{2} \mathrm{x} \cos \omega \theta\right\}$

We can deduce from the equation (3.12) and (3.9) that

$\frac{1}{\pi} \int \frac{\mathrm{r}(\mathrm{u})}{\mathrm{L}-\mathrm{u})} \mathrm{du}=-\frac{\mathrm{b}(\xi)}{\mathrm{a}(\xi)} \mathrm{s}_{1}(\mathrm{x}), \mathrm{a}<\mathrm{x}<\mathrm{b}$

It is simple matter to show that for $\mathrm{x}>\mathrm{b}$,

$\Lambda^{+}(\mathrm{x})=\frac{\mathrm{if} \mathrm{f}_{0}}{2 \mathrm{a}(\xi)}-\frac{\mathrm{f}_{0}}{2 \mathrm{a}(\xi)}\left(\left(\mathrm{x}^{2}-\mathrm{a}^{2}\right)\left(\mathrm{x}^{2}-\mathrm{b}^{2}\right)\right)^{-1 / 2}\left\{\left(\mathrm{x}^{2}+\right.\right.$

$\left.\left.\mathrm{h}_{2}^{1}\right) \cos \omega \theta_{2} \mathrm{~h}_{2}^{1} \mathrm{x} \sin \omega \theta_{2}\right)-\left(\left(\mathrm{x}^{2}+\mathrm{h}_{2}^{1}\right) \sin \omega \theta_{2}\right.$

$\left.\left.\mathrm{h}_{2}^{1} \mathrm{x} \cos \omega \theta_{2}\right)\right\}$

and hence, for $\mathrm{x}>\mathrm{b}$

$\sigma_{\text {yy }}(\mathrm{x}, \quad 0+)=-\mathrm{P}_{0}(1+\mathrm{i})\left(1-\left\{\left(\mathrm{x}^{2}-\mathrm{a}^{2}\right)\left(\mathrm{x}^{2}-\right.\right.\right.$ $\left.\left.\left.b^{2}\right)\right\}^{-1 / 2}\left\{\left(x^{2}+h_{2}^{1}\right) \cos \omega \theta_{2}-h_{2}^{1} x \sin \omega \theta_{2}\right\}\right)$

$\sigma_{\mathrm{xy}}(\mathrm{x}, 0+)=-\mathrm{P}_{0}(1+\mathrm{i})\left(\left\{\left(\mathrm{x}^{2}-\mathrm{a}^{2}\right)\left(\mathrm{x}^{2}-\mathrm{b}^{2}\right)\right)^{-1 / 2}\left\{\left(\mathrm{x}^{2}+\right.\right.\right.$ $\left.\left.\left.\mathrm{h}_{2}^{1}\right) \sin \omega \theta_{2}-\mathrm{h}_{2}^{1} \mathrm{x} \cos \omega \theta_{2}\right\}\right)$

$\mathrm{m}_{\text {фy }}(\mathrm{x}, \quad 0+)=-\mathrm{P}_{0}(1+\mathrm{i})\left(1-\left\{\left(\mathrm{x}^{2}-\mathrm{a}^{2}\right)\left(\mathrm{x}^{2}-\right.\right.\right.$ $\left.\left.\left.b^{2}\right)\right)^{-1 / 2}\left\{\left(x^{2}+h_{2}^{1}\right) \sin \omega \theta_{2}+h_{1}^{2} x \cos \omega \theta_{2}\right\}\right)$

where

$\theta_{2}=\log \left\{\frac{(x-a)(x+b)}{(x+a)(x-b)}\right\}$

We see from (3.17), (3.18) and (3.19) that as $\mathrm{x} \rightarrow \infty$

$\sigma_{\text {yy }}(\mathrm{x}, 0+)=-\mathrm{P}_{0}(1+\mathrm{i}) 0\left(\mathrm{x}^{-1}\right)$

$\sigma_{\mathrm{xy}}(\mathrm{x}, 0+)=\mathrm{P}_{0}(1+\mathrm{i})\left\{2 \omega(\mathrm{b}-1)+\mathrm{h}_{1}^{2}\right\}\left(\mathrm{x}^{-1}\right)+0\left(\mathrm{x}^{-1}\right)$

$\mathrm{m}_{\phi y}(\mathrm{x}, 0+)=\mathrm{P}_{0}(1+\mathrm{i}) 0\left(\mathrm{x}^{-1}\right)$

Hence, it follows that the condition $\sigma_{\mathrm{yy}}(\mathrm{x}, 0+)=$ $0\left(\mathrm{x}-{ }^{1}\right)$ and $\mathrm{m}_{\phi \mathrm{y}}(\mathrm{x}, 0+)=0\left(\mathrm{x}-{ }^{1}\right)$ as $\mathrm{x} \rightarrow \infty$ is automatically satisfied while that $\sigma_{\mathrm{yy}}(\mathrm{x}, 0+)=0\left(\mathrm{x}-{ }^{1}\right)$ as $x \rightarrow \infty$ will only be satisfied if we choose $h_{2}^{1}=-2 \omega(b-$ a). Thus it only remains to determine the constant $h_{2}^{1}$. This 
is determined from the condition that at the end point $\mathrm{x}=\mathrm{a}$, $\mathrm{u}_{\mathrm{y}}(\mathrm{a}, 0+)=0=\mathrm{u}_{\mathrm{y}}(\mathrm{a}, 0-)$. From (2.10), (2.19) (3.9) and (3.15) we see that

$\mathrm{u}_{\mathrm{y}}(\mathrm{x}, 0+)=\frac{\mathrm{s}_{1}(\mathrm{x}) \cdot \mathrm{c}}{\mathrm{a}(\xi) \cdot \mathrm{a}^{2}}, \mathrm{a}<\mathrm{x}<\mathrm{b}$

$\mathrm{u}_{\mathrm{y}}(\mathrm{x}, 0-)=\frac{\mathrm{s}_{1}(\mathrm{x}) \cdot \mathrm{D}}{\mathrm{a}(\xi) \cdot \mathrm{a}^{2}}, \mathrm{a}<\mathrm{x}<\mathrm{b}$

where $\mathrm{C}$ and $\mathrm{D}$ can be calculated.

Therefore, we have condition

$$
s_{1}(a)=\int_{a}^{b} s_{1}(u) d u=0
$$

This gives

$h_{2}^{1}=\frac{-I_{2}+2 \omega(b-a) I_{1}}{I_{0}}$

Where

$$
\mathrm{I}_{0} \quad=\quad \int_{\mathrm{a}}^{\mathrm{b}} \frac{\cos \omega \theta \mathrm{du}}{\sqrt{\left(\mathrm{u}^{2}-\mathrm{a}^{2}\right)\left(\mathrm{b}^{2}-\mathrm{u}^{2}\right)}}=\frac{\pi}{(\mathrm{a}+\mathrm{b}) \cosh \pi \omega} 2 \mathrm{~F}_{1}(1 / 2+
$$$$
\left.\mathrm{i} \omega, 1 / 2,1, \mathrm{z}^{2}\right)
$$$$
I_{1}=\int_{a}^{b} \frac{u \sin \omega \theta d u}{\sqrt{\left(u^{2}-a^{2}\right)\left(b^{2}-u^{2}\right)}}=\frac{\pi \omega z}{\cosh \pi \omega} F_{3}(1 / 2+i \omega, 1 / 2-i \omega, 1 / 2-
$$$$
i \omega, 1 / 2+i \omega, 2, z-z)
$$$$
\mathrm{I}_{2}=\int_{\mathrm{a}}^{\mathrm{b}} \frac{\mathrm{u}^{2} \cos \omega \theta \mathrm{du}}{\sqrt{\left(\mathrm{u}^{2}-\mathrm{a}^{2}\right)\left(\mathrm{b}^{2}-\mathrm{u}^{2}\right)}}=\frac{\pi \mathrm{a}^{2}}{(\mathrm{a}+\mathrm{b}) \cosh \pi \omega} \mathrm{F}_{3}(1 / 2-\mathrm{i} \omega, 1 / 2+
$$
$i \omega, 1 / 2+i \omega, 1 / 2-i \omega, 1, z-z)$

$+\frac{\pi(b-a)}{2 \cosh \pi \omega} F_{3}(1 / 2-i \omega, 1 / 2+i \omega, 1 / 2+i \omega, 1 / 2+i \omega, 2, z-z)$

$-\frac{\pi(b-a)^{2}}{4(b+a) \cosh \pi \omega} \sum_{p=0}^{\infty} \sum_{\mathrm{q}=0}^{\infty} \frac{(1 / 2-i \omega)_{\mathrm{p}}(1 / 2+i \omega)_{\mathrm{q}}(1 / 2+\mathrm{i} \omega)_{\mathrm{p}}(1 / 2-i \omega)_{\mathrm{q}}}{3 ! \mathrm{q} !(3)_{\mathrm{p}+\mathrm{q}}}$

$\cdot(2 \mathrm{q}+1)(\mathrm{q}-\mathrm{p}) \mathrm{z}^{\mathrm{p}}(-\mathrm{z})^{\mathrm{p}}$

and $\mathrm{F}_{3}$ is hypergeometric function of two variables defined in (112, p 274).
For $\mathrm{x}>\mathrm{b}$ stresses are given by

$\sigma_{\mathrm{yy}}(\mathrm{x}, 0+)=\sigma_{\mathrm{yy}}(\mathrm{x}, 0-)=-\mathrm{P}_{0}(1+\mathrm{i})\left(1-\left\{\left(\mathrm{x}^{2}-\right.\right.\right.$ $\left.\left.a^{2}\right)\left(x^{2}-b^{2}\right)\right\}^{-1 / 2}\left\{\left(x^{2}+h_{2}^{1}\right) \cos \omega \theta_{2}+2 \omega(b-\right.$

a) $\left.\left.x \sin \omega \theta_{2}\right\}\right)(3.23)$

$\sigma_{\mathrm{xy}}(\mathrm{x}, 0+)=\sigma_{\mathrm{xy}}(\mathrm{x}, 0-)=\mathrm{P}_{0}(1+\mathrm{i})\left(\left\{\left(\mathrm{x}^{2}-\mathrm{a}^{2}\right)\left(\mathrm{x}^{2}-\right.\right.\right.$ $\left.\left.\left.\mathrm{b}^{2}\right)\right\}^{-1 / 2}\left\{\left(\mathrm{x}^{2}+\mathrm{h}_{2}^{1}\right) \sin \omega \theta_{2}-2 \omega(\mathrm{b}-\mathrm{a}) \mathrm{x} \cos \omega \theta_{2}\right\}\right)$

$\mathrm{m}_{\phi \mathrm{y}}(\mathrm{x}, 0+)=\mathrm{m}_{\phi \mathrm{y}}(\mathrm{x}, 0-)=\mathrm{P}_{0}(1+\mathrm{i})\left(-1+\left\{\left(\mathrm{x}^{2}-\right.\right.\right.$ $\left.\left.a^{2}\right)\left(x^{2}-b^{2}\right)\right\}^{-1 / 2}\left\{\left(x^{2}+h_{2}^{1}\right) \cos \omega \theta_{2}-2 \omega(b-\right.$

a) $\left.\left.x \sin \omega \theta_{2}\right\}\right)$

The stresses for $0<\mathrm{x}<\mathrm{a}$ are given by

$\sigma_{\mathrm{yy}}(\mathrm{x}, 0+)=\sigma_{\mathrm{yy}}(\mathrm{x}, 0-)=\mathrm{P}_{0}(1+\mathrm{i})\left(1+\left\{\left(\mathrm{a}^{2}-\mathrm{x}^{2}\right)\left(\mathrm{b}^{2}-\right.\right.\right.$ $\left.\left.\mathrm{x}^{2}\right)\right\}^{-1 / 2}$

$\left\{\left(x^{2}+h_{2}^{1}\right) \cos \omega \theta_{2}+2 \omega(b-a) x \sin \omega \theta_{1}\right\}(3.26)$

$\sigma_{\mathrm{xy}}(\mathrm{x}, 0+)=\sigma_{\mathrm{xy}}(\mathrm{x}, 0-)=\mathrm{P}_{0}(1+\mathrm{i})\left\{\left(\mathrm{a}^{2}-\mathrm{x}^{2}\right)\left(\mathrm{b}^{2}-\right.\right.$ $\left.\left.x^{2}\right)\right\}^{-1 / 2}$

$\left\{\left(x^{2}+h_{2}^{1}\right) \sin \omega \theta_{1}-2 \omega(b-a) x \cos \omega \theta_{1}\right\}(3.27)$

$\mathrm{m}_{\phi \mathrm{y}}(\mathrm{x}, 0+)=\mathrm{m}_{\phi \mathrm{y}}(\mathrm{x}, 0-)=\mathrm{P}_{0}(1+\mathrm{i})\left(-1+\left\{\left(\mathrm{a}^{2}-\right.\right.\right.$ $\left.\left.x^{2}\right)\left(b^{2}-x^{2}\right)\right\}^{-1 / 2}\left\{\left(x^{2}+h_{2}^{1}\right) \cos \omega \theta_{1}-2 \omega(b-\right.$

a) $\left.x \sin \omega \theta_{1}\right\}$

where

$\theta_{1}=\log \left\{\frac{(a-x)(b-x)}{(a+x)(b+x)}\right\}$

$\mathrm{h}_{2}^{1}$ can be calculated from $(3.22)$

\section{The Stress Intensity Factors}

If $\mathrm{N}_{1 \mathrm{~b}}, \mathrm{~N}_{2 \mathrm{~b}}$ and $\mathrm{N}_{3 \mathrm{~b}}$ are the normal and shear stress intensity factors at the crack tip $x=b$ then

$$
\begin{aligned}
& \mathrm{N}_{1 \mathrm{~b}}=\lim _{x \rightarrow b}\left[(\mathrm{x}-\mathrm{b})^{1 / 2} \sigma_{\mathrm{yy}}(\mathrm{x}, 0+)\right] \\
& \mathrm{N}_{2 \mathrm{~b}}=\lim _{\square \rightarrow \square}\left[(\mathrm{x}-\mathrm{b})^{1 / 2} \sigma_{\mathrm{xy}}(\mathrm{x}, 0+)\right]
\end{aligned}
$$


$\mathrm{N}_{3 \mathrm{~b}}=\lim _{\square \rightarrow \square}\left[(\mathrm{x}-\mathrm{b})^{1 / 2} \mathrm{~m}_{\phi \mathrm{y}}(\mathrm{x}, 0+)\right]$

then from the equations (3.23), (3.24) and (3.25), we get

$$
\begin{aligned}
\mathrm{N}_{1 \mathrm{~b}}+\mathrm{N}_{2 \mathrm{~b}}+\mathrm{N}_{3 \mathrm{~b}} & =\frac{\mathrm{p}_{0}(1+\mathrm{i})}{2 \mathrm{~b}\left(\mathrm{~b}^{2}-\mathrm{a}^{2}\right)}\left\{4 \omega \mathrm{b}^{2}(\mathrm{~b}-\mathrm{a})^{2}+\left(\mathrm{b}^{2}\right.\right. \\
& \left.\left.+\mathrm{h}_{2}^{1}\right)\right\}
\end{aligned}
$$

Similarly, the normal and shear stress intensity factor at the crack tip $\mathrm{x}=\mathrm{a}$ are given by

$\mathrm{N}_{1 \mathrm{a}}+\mathrm{N}_{2 \mathrm{a}}+\mathrm{N}_{3 \mathrm{a}}=\frac{\mathrm{p}_{0}(1+\mathrm{i})}{2 \mathrm{~b}\left(\mathrm{~b}^{2}-\mathrm{a}^{2}\right)}\left\{\omega \mathrm{a}^{2}(\mathrm{~b}-\mathrm{a})^{2}+\left(\mathrm{a}^{2}+\mathrm{h}_{2}^{1}\right)\right\}$

\section{RESULTS AND DISCUSSION}

In this paper, we consider a pair of coplanar Barenblatt cracks at the interface of the two bonded dissimilar micropolar elastic half planes. The components of stress and displacement have been calculated. The problem is reduced to the system of simultaneous dual integral equations which are further transformed to a Riemann boundary value problem. Calculations for evaluating the stress intensity factors at the crack tip are derived.

\section{ACKNOWLEDGEMENT}

I would like to acknowledge the help received from Dr. P.K. Tripathi, the department of Mathematics, CSJMU Kanpur.

\section{REFERENCES}

Barenblatt G.I., 1962. The mathematical theory of equilibrium cracks in brittle fracture', Advances in Applied Mechancis, 7: 55-129.

Burniston E.E. and Gurely W.Q., 1973. 'The effect of partial closure on the stress intensity factor of a Griffith crack opened by a parabolic pressure distribution', Int. J. Fracture, 9: 9-12.

Eedelyi A., 1954. Higher Transcendental Functions, Megraw Hill: 1.

Green A.E. and Zerna W., 1960. 'Theoretical Elasticity', Oxford.

Lord M.W., Shulman Y. and Mech J., 1967. Phys. Solids, 15: 229.

Sneddon I.N., 1969. 'The distribution of surface stress necessary to produce a Griffith crack of prescribed shape', Int. J. Engg. Sci., 7: 875882.

Sneddon I.N. and Lowengrub, M., 1969. 'Crack problems in the classical theory of elasticity, SIAM, Monograph Wiley.

Takao and Suharam T., 1987. A fibre reinforced matrix containing a penny-shaped crack under mode III loading condition', Int. J. Engg. Sci., 25: 855-896.

Thresher and Smith F.W., 1973. 'The partially closed Griffith crack', Int. J. Fracture, 9: 33-41. 